\title{
Chapter 7 \\ Equatorin-Related Subcellular and Molecular Events During Sperm Priming for Fertilization in Mice
}

\author{
Chizuru Ito, Kenji Yamatoya, and Kiyotaka Toshimori
}

\begin{abstract}
Spermatozoa must undergo a priming process that renders them competent for fertilization. This priming process involves the initiation of acrosomal exocytosis, remodeling of the acrosomal substructure, and biochemical modification of related molecules. However, the mechanism underlying sperm priming process has remains unclear because of the number of molecules involved in sperm capacitation and the ensuing acrosome reaction (acrosomal exocytosis). Here, we focus on the acrosomal type 1 membrane protein equatorin and the related subcellular and molecular events that occur during the sperm priming process in mice.
\end{abstract}

Keywords Acrosome $\bullet$ Acrosome reaction $\bullet$ Equatorin $\bullet$ Fertilization

\subsection{Introduction}

Sperm priming involves the specific subcellular and molecular changes occurring in capacitated spermatozoa that are inevitable for sperm-egg fusion (Yanagimachi 1994; Toshimori 2009). The priming events occur at the periacrosomal plasma membrane of spermatozoa that approach or arrive at the zona pellucida, and the changes appear to continue in a fertilizing spermatozoon that penetrates into the perivitelline space. However, the underlying mechanism remains unresolved

\footnotetext{
C. Ito $\bullet \mathrm{K}$. Toshimori $(\bowtie)$

Department of Reproductive Biology and Medicine, Graduate School of Medicine,

Chiba University, Chiba 260-8670, Japan

e-mail: ktoshi@faculty.chiba-u.jp

K. Yamatoya

Department of Reproductive Biology and Medicine, Graduate School of Medicine,

Chiba University, Chiba 260-8670, Japan

Biomedical Research Center, Chiba University, Chiba 260-8670, Japan
} 
because the priming process is rapid and complicated and because the precise localization and chemical nature of the molecules involved are still unclear. As the initial changes are thought to initiate in the apical region of the anterior acrosome (Yanagimachi 1994; Eddy 2006; Toshimori 2009), the essential sperm molecules involved in the priming process are presumed to be localized at the plasma membrane, in the periacrosomal space, on the outer acrosomal membrane, and in the anterior acrosome. However, there are unknown pathway linkages between the apical region and other sperm domains during capacitation (Buffone et al. 2012). To date, proteins anchored to the plasma membrane that are important for mediating the capacitation include calcium-related proteins, such as CatSper1, CatSper 2, and PMCA (Eddy 2006). SNARE regulatory proteins, such as $\alpha$-SNAP, NSF, rab3a (small GTPase), and synaptotagmins, are detected around the acrosomal region, suggesting their involvement in the regulation of acrosomal exocytosis (Tomes et al. 2002; Michaut et al. 2001; Yunes et al. 2002). The outer acrosomal membraneanchored proteins could be involved in the priming process, including the signaling pathway to activate internal molecules, and could also function as components of the platform to maintain the acrosomal structure and other crucial fertilization events. These proteins include ADAM3/cyritestin (Forsbach and Heinlein 1998), ZPBP1/SP38/IAM38 (Yu et al. 2006; Ferrer et al. 2012), SPACA1/SAMP32 (Hao et al. 2002; Ferrer et al. 2012), SPACA4/SAMP14 (Shetty et al. 2003), OBF13/ Izumo1 (Okabe et al. 1987; Inoue et al. 2005), SPESP1/ESP (Wolkowicz et al. 2003), and MN9/equatorin (Toshimori et al. 1992, 1998). Because the proteins proposed to be essential for sperm-egg fusion, sperm Izumo1 (Inoue et al. 2005) and egg CD9 (Kaji et al. 2000; Le Naour et al. 2000; Miyado et al. 2000), are discussed in other chapters; here, we focus on the nature of the acrosomal protein equatorin (Eqtn) and its related subcellular and molecular events during the sperm priming process in mice.

\subsection{Equatorin and Its Chemical Nature}

Equatorin was first detected as an MN9 antigen in the mouse, rat, hamster, and human mature spermatozoa using the mouse monoclonal antibody MN9 (Toshimori et al. 1992). Equatorin is composed of a complex of $48-\mathrm{kDa}$ and $38-\mathrm{kDa}$ proteins located in the acrosome. The antigen was renamed "equatorin" because the MN9 antibody showed a strong affinity for the equatorial segment and the MN9 antigen was found to be enriched in the equatorial segment (Toshimori et al. 1998). Purified MN9 antibody suppresses the events that occur from acrosome reaction to egg activation under in vitro (Toshimori et al. 1998) and in vivo conditions (Yoshinaga et al. 2001). The MN9 antigen is relocated from the acrosome to the plasma membrane over the equatorial segment during the acrosome reaction (Manandhar and Toshimori 2003). Equatorin was purified by immunoprecipitation using the MN9 antibody, and purified equatorin was identified by LC-MS/MS analysis (Fig. 7.1) (Yamatoya et al. 2009). A Mascot search revealed a single significant candidate, 


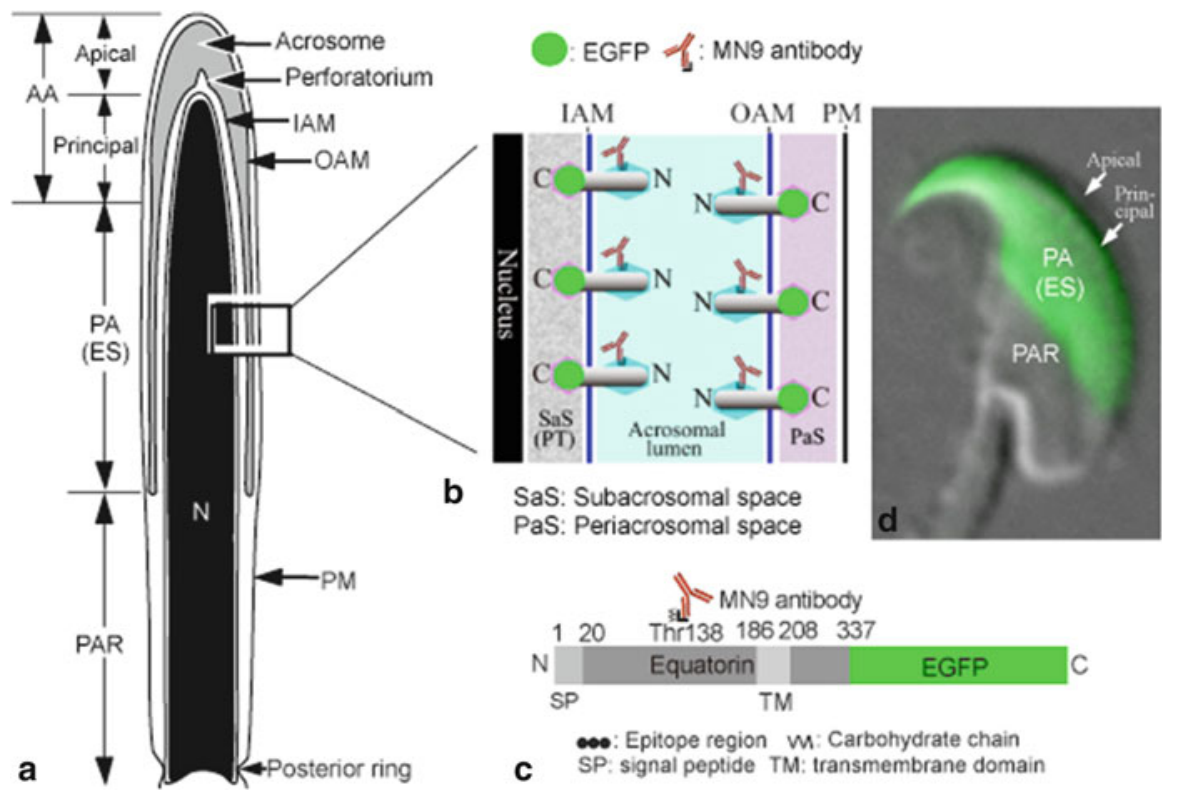

Fig. 7.1 Sperm domains, localization of equatorin, topology of the epitope recognized by the MN9 antibody and Eqtn-EGFP, and a generated Eqtn-EGFP transgenic mouse spermatozoon. (a, b) The MN9 antibody recognizes the N-terminus in the acrosomal lumen, whereas EGFP is present at the C-terminus side in both the periacrosomal and subacrosomal spaces. (c, d) The molecular structure of the mouse Eqtn-EGFP chimeric protein (c) used to generate the Eqtn-EGFP transgenic spermatozoa (d). The conserved domains are 1-20 amino acids (aa) for the potential signal peptide (SP) and 186-208 aa for the transmembrane (TM) domain; the epitope for the MN9 antibody is present in the region containing a carbohydrate chain branched from the threonine at aa 138 (Thr138). AA anterior acrosome, ES equatorial segment, IAM inner acrosomal membrane, $O A M$ outer acrosomal membrane, $P A$ posterior acrosome, $P A R$ postacrosomal region, $P M$ plasma membrane

4930579C15Rik, the human orthologue of which is reported to be frequently deleted in cancer (Ruiz et al. 2000). The equatorin gene and its chemical nature were clarified with the aid of recently developed techniques for carbohydrates, such as the Pro-Q Emerald 300 glycoprotein gel staining kit and SYPRO Ruby protein gel stain. Equatorin is a highly glycosylated and sialylated protein, an $\mathrm{N}, \mathrm{O}$ sialoglycoprotein, and it is insoluble in mild detergents. There are long and short forms of equatorin encoded by the Eqtn gene, which is located on mouse chromosome 4 (the EQTN gene on human chromosome 9, 9p21). The core protein size is approximately $27 \mathrm{kDa}$, and the $O$-sialylated carbohydrate region branching from the threonine 138 is involved in the epitope for the MN9 antibody (Fig. 7.2). Equatorin is also called Afaf (Li et al. 2006) or C9orf11 (Ruiz et al. 2000).

Information on the equatorin epitope region recognized by the MN9 antibody is based on Galnt3 gene deletion experiments. Galnt3-null mouse homozygotic testicular germ cells exhibit drastically reduced reactivity with the MN9 antibody, and the mice are infertile because of oligoashthenoteratozoospermia (Miyazaki et al. 2013). 


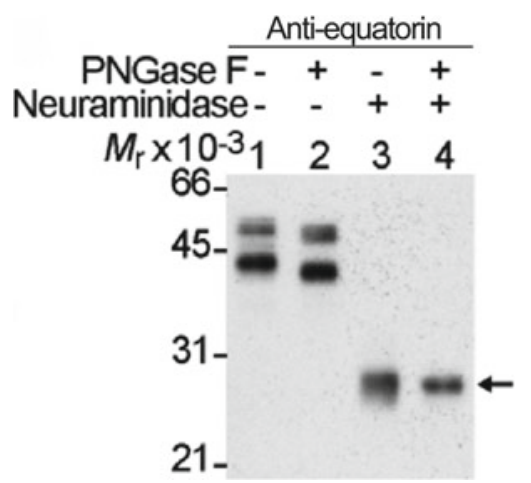

Fig. 7.2 Glycosylation status of equatorin by mobility shift assay using glycosidase treatment. Lanes 1-4: cauda sperm extract analyzed by Western blotting (15\% gel) with the MN9 antibody. Lane 1, without glycosidase treatment. Lane 2, treatment with PNGase F only. Lane 3, treatment with neuraminidase only. Lane 4, treatment with PNGase F and neuraminidase. Mobility shifts are observed with both the PNGase and neuraminidase treatments, but the MN9 antigenicity remains on the molecule at approximately $27 \mathrm{kDa}$ (arrow). (Modified from Yamatoya et al. 2009)

In these mutant mice, spermatozoa are rare in the cauda epididymides, most of which exhibit deformed rounded heads. Galnt3 is a GalNAc transferase family protein involved in the initiation of mucin-type $O$-glycosylation (Bennett et al. 1996). The VVA (Vicia villosa agglutinin) lectin, which can recognize the Tn antigen (GalNAc-O-Ser/Thr) generated by GalNac transferase, also recognizes the acrosomal regions of spermatids and spermatozoa, whereas VVA binding is drastically reduced in Galnt3-null mice (Miyazaki et al. 2013). Thus, GlcNAc $\beta 1-3-G a l N A c \alpha 1-S e r / T h r$ mediated by Galnt3 (GalNAc transferase) is involved in the formation of the equatorin epitope region recognized by the MN9 antibody.

\subsection{Expression and Molecular Size of Equatorin in the Testis}

When examined by in situ hybridization, mouse Eqtn mRNA is specifically expressed in early- to mid-round spermatids (step 1-6) and decreases in late-round (steps 7-8) spermatids (Ito et al. 2013). The molecular size of mouse equatorin is approximately $65 \mathrm{kDa}$ in testicular germ cells (Ito et al. 2013), but the size decreases to $40-50 \mathrm{kDa}$ in cauda epididymal spermatozoa when examined by Western blotting (Yoshida et al. 2010).

\subsection{Localization of Equatorin in Mature Spermatozoa}

Equatorin is anchored to the acrosomal membrane (type 1 membrane protein) in mature spermatozoa, but the amount in the acrosomal membrane varies among the domains. When examined by immunogold electron microscopy, the immunogold 
particles are abundant on the inner acrosomal membrane in the principal region and equatorial segment (Ito et al. 2013). However, on the outer acrosomal membrane, the gold particles are depleted in the principal region but abundant at the equatorial segment, whereas gold particles are not detected on the outer acrosomal membrane in the apical region, suggesting that equatorin is absent or very poor on the outer acrosomal membrane in the apical region. In addition, electron microscopic data suggest that equatorin is embedded in electron-dense acrosomal matrix substances, locations where immunogold particles are generally 5-70 $\mathrm{nm}$ away from the acrosomal membranes, with the N-terminus in the acrosomal lumen (Ito et al. 2013). Such three-dimensional structures suggest that the $\mathrm{N}$-terminus of equatorin is embedded in the complex of the inner acrosomal membrane and acrosomal matrix (CIMAM) and the complex of the outer acrosomal membrane and acrosomal matrix (COMAM) (Toshimori 2011; Ito et al. 2013). Similarly, the C-terminus of equatorin appears to associate with the perinuclear theca substances in the subacrosomal space and with the periacrosomal substances in the periacrosomal space.

These lines of subcellular evidence provide some clues to propose the function of equatorin in spermatozoa, as deficiency in the acrosomal membrane-binding matrix protein SPESP1 (ESP) causes embrittlement of the equatorial segment but increases the expression of equatorin (Fujihara et al. 2012). Because SPESP-1 is known to be localized to the equatorial segment of ejaculated human sperm (Wolkowicz et al. 2003), SPESP1 may work redundantly with equatorin. Antisera raised against recombinant SPESP-1 inhibit the binding and fusion of human sperm to the hamster eggs (Wolkowicz et al. 2008), but SPESP-1 cannot be detected by a rabbit anti-mouse SPESP1 polyclonal antiserum that was produced by immunization with mouse SPESP1 polypeptide (MYGSNVFPEGRTSD) after the acrosome reaction (Fujihara et al. 2012). SPESP-1deficient mice are fertile, although Spesp1+/- and Spesp1-/- spermatozoa have a lower fusing ability compared to wild-type spermatozoa (Fujihara et al. 2010). The protein(s) associated with equatorin have not been identified to date.

\subsection{Behavior of Equatorin During the Acrosome Reaction}

\subsubsection{Before and the Very Initial Stage of the Acrosome Reaction}

Equatorin is not detected before or at the very initial stage of the acrosome reaction by indirect immunofluorescence (IIF) microscopy using the MN9 antibody because the antibody cannot reach the epitope region on the N-terminus of equatorin, which is in the acrosomal lumen. However, as the acrosome reaction proceeds, the epitope region is gradually exposed at the area near the principal regions and becomes detected by the MN9 antibody (Fig. 7.3). When examined by IIF microscopy using an antibody against Izumo1 (\#125), the MN9 epitope region appears later than Izumo1 (unpublished data), which is localized on the outer and inner acrosomal membranes of the apical segment (Inoue et al. 2005; Satouh et al. 2012). 


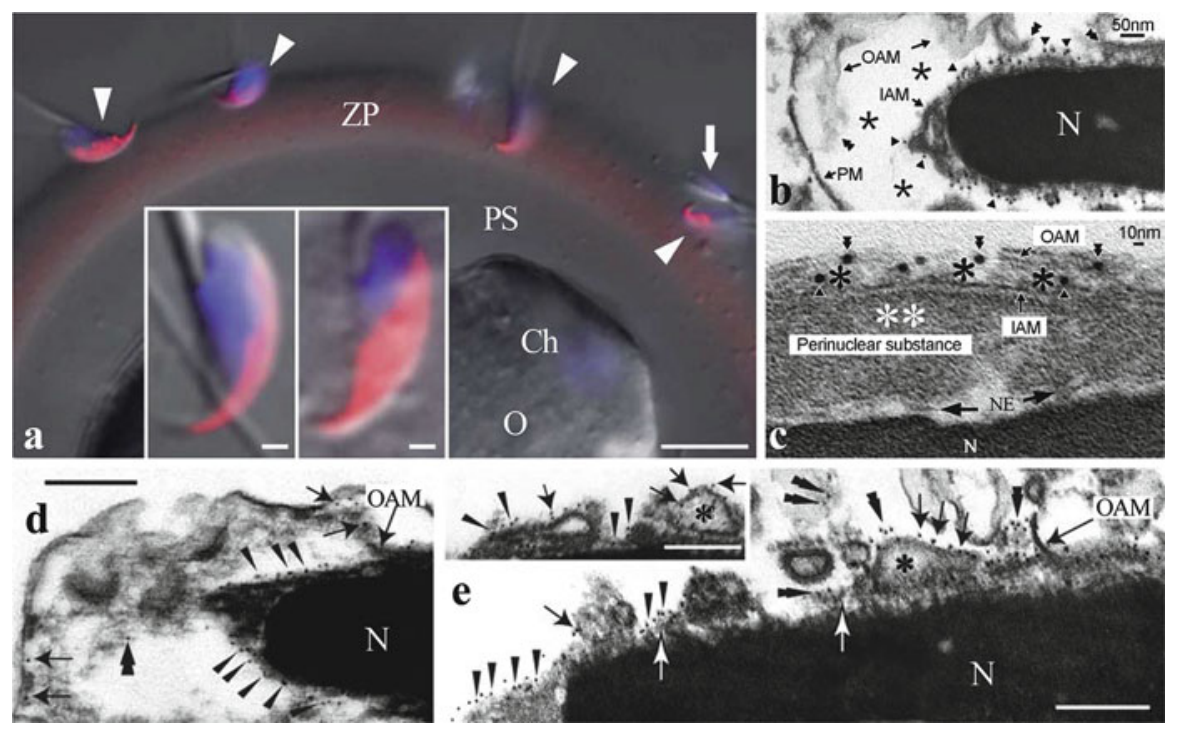

Fig. 7.3 Subcellular behavior of equatorin during the acrosome reaction. Indirect immunofluorescence with the MN9 antibody (a) and immunogold electron microscopy with the MN9 antibody (b-e). (a) Various stages of the acrosome reaction are found in spermatozoa that are attached to the zona pellucida (arrowheads). An acrosome-intact spermatozoon is indicated with an arrow. Left and right insets: High-magnification images of different types of MN9-immunostaining patterns. $C h$ chromosome, $O$ ooplasm, $P S$ perivitelline space, $Z P$ zona pellucida. MN9 red, nucleus or chromosome blue (Hoechst). Bars a $10 \mu \mathrm{m}$; insets $1 \mu \mathrm{m}$. (b, c) Immunogold particles (10-nm gold particles, arrowheads) are abundant on the inner acrosomal membrane facing the acrosome lumen, as indicated with asterisks, but depleted (no gold particles in this photograph) on the outer acrosomal membrane in the anterior acrosomal region before or at the very initial stage of the acrosome reaction; gold particles are not present on the plasma membrane. In the posterior acrosome (equatorial segment), the gold particles are present on both the inner (arrowheads) and outer (double arrowheads) acrosomal membranes; the particles appear to associate with the electron-dense matrix substances facing the narrowed internal lumen $(*)$. Electron-dense perinuclear substances (** in this figure; Ito et al. 2013) are found in the space between the inner acrosomal membrane and the nuclear envelope $(N E)$. (d, e) Early stage (d) and advanced stage (e) of the acrosome reaction. Immunogold particles $(5 \mathrm{~nm}$ ) are dense on the inner acrosomal membrane (arrowheads) and weak on the outer acrosomal membrane (arrows) and in the amorphous substance (double arrowhead). In the advanced stage (e), some gold particles are found in association with the hybrid vesicles (asterisks) formed by the plasma membrane and outer acrosomal membrane (arrows in $\mathbf{e}$ and inset). Some other gold particles are present on the inner acrosomal membrane (arrowheads). Bar e $200 \mathrm{~nm}$. IAM inner acrosomal membrane, $N$ nucleus, $O A M$ outer acrosomal membrane, $P M$ plasma membrane. (a-c and d, e reproduced with slight modifications from Yoshida et al. 2010) and Yamatoya et al. 2009, respectively)

\subsubsection{Early to Middle Stages of the Acrosome Reaction}

Some amount of equatorin is relocated on the surface of the plasma membrane over the equatorial segment but not to the plasma membrane over the postacrosomal region (Yoshida et al. 2010). This fact suggests that the epitope region recognized 

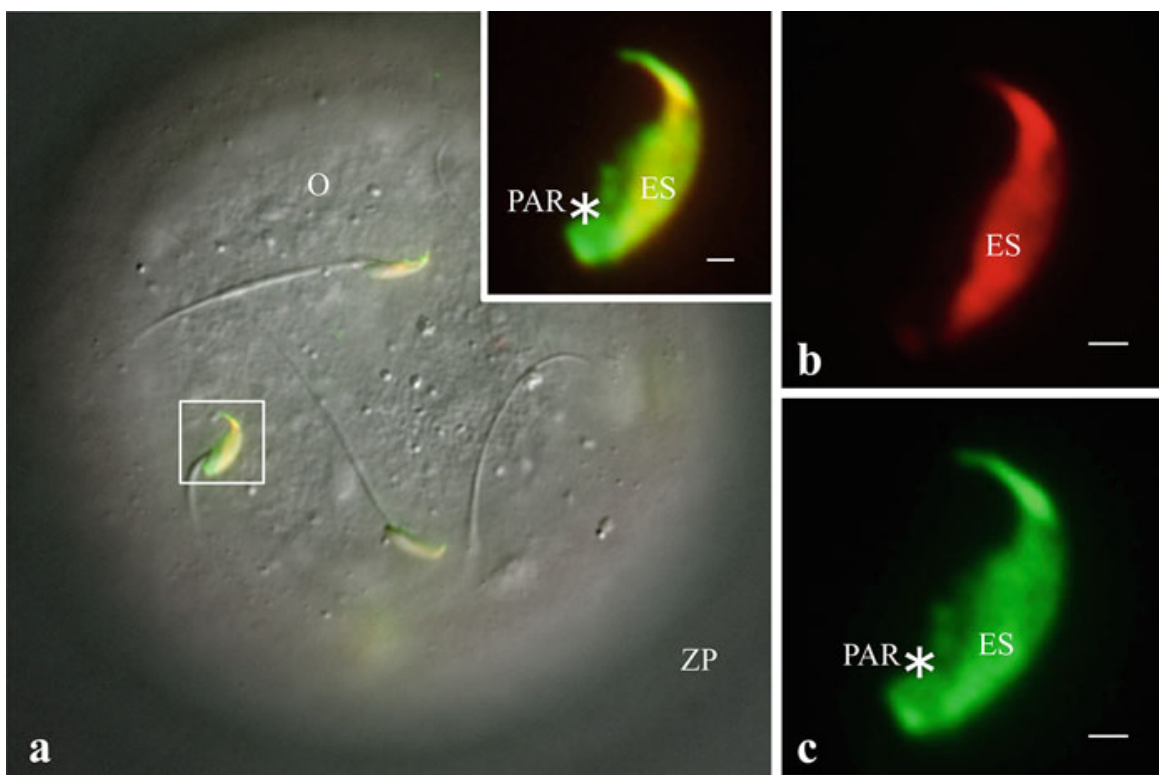

Fig. 7.4 Localization of equatorin in the perivitelline spermatozoa. Indirect immunofluorescence double staining with the anti-equatorin antibody MN9 antibody (red) and anti-Izumo1 antibody (green). Perivitelline spermatozoa accumulated in the perivitelline space in CD9-null eggs are the subjects of the immunofluorescence study. The square area at the lower magnification (a) of the merged DIC image is enlarged in the insets of $\mathbf{a}, \mathbf{b}$, and $\mathbf{c}$. Equatorin is localized at the equatorial segment region ( $E S$ in inset and $\mathbf{b}$ ), whereas Izumol is found at both the equatorial segment and postacrosomal region (* in c). $O$ oocyte surface, $Z P$ zona pellucida. Bar $1 \mathrm{~m}$

by the MN9 antibody has a specific affinity for the equatorial plasma membrane; however, a sufficient amount of equatorin remains in the acrosome-reacted spermatozoa. Immunofluorescence double-staining data using the anti-Izumo1 antibody and anti-MN9 antibody suggest that Izumo1 can spread rapidly and widely to the postacrosomal region in the acrosome-reacting spermatozoa, whereas the equatorin epitope region recognized by MN9 antibody remains confined to the anterior acrosomal region (unpublished data). The molecular size of equatorin appears to be reduced to $35 \mathrm{kDa}$ at the end of the acrosome reaction (Yoshida et al. 2010).

\subsubsection{Advanced Stage and After the Acrosome Reaction}

A sufficient amount of equatorin remains in the acrosomal membranes in the principal region and at the equatorial segment in the advanced stage of the acrosome reaction; this pattern is similar in a fertilizing spermatozoon that reaches the perivitelline space (i.e., perivitelline spermatozoon). In fact, the perivitelline spermatozoa accumulated in the perivitelline space of CD9-deficient eggs display both equatorin and Izumo1 (Fig. 7.4). 
The equatorial segment is stabilized by the extensive scaffolding network that connects the outer acrosomal membrane to the inner acrosomal membrane (Fawcett 1975), a site thought to contain many enzymes and matrix molecules (Yanagimachi 1994; Eddy 2006; Toshimori 2009). The equatorin protein that is stably embedded in this network is carried into the ooplasm where associated enzymes and matrix molecules are fully released. A study investigating this process by employing Eqtn-EGFP transgenic mouse spermatozoa is currently being performed in our laboratory.

\subsection{Possible Roles of Equatorin}

At present, there are several lines of evidence invoked to propose the roles for equatorin. First, the evidence that the protein is produced and distributed in the acrosomal membrane in early spermatids and is retained in mature spermatozoa (Figs. 7.1, 7.3, 7.5) suggests that equatorin serves as a platform component to stabilize the acrosomal membrane and to maintain the shape of the acrosome. This role is presumed to continue until the sperm-egg interaction stage. Within this context, the nature of the branching sialylated carbohydrate chains (MN9 epitope region) on the threonine 138 (Figs. 7.1, 7.2) at the N-terminus will be important because the protein is maintained in the acidic environment of the acrosome lumen before the acrosome reaction, whereas the C-terminus is under neutral conditions, that is, in the periacrosomal space or in the perinuclear space. Equatorin may also function to anchor other molecules, although supporting evidence is not available thus far. Second, some amount of the equatorin that is relocated to the equatorial segment plasma membrane (Fig. 7.4) may play additional roles, as previously suggested by the findings that the MN9 antibody could suppress the events from acrosome reaction to egg activation under in vitro and in vivo conditions (Toshimori et al. 1998; Yoshinaga et al. 2001).

\subsection{Perspective}

Our next purpose is to determine the precise role of equatorin in the course of fertilization process and during spermatogenesis. To pursue this purpose, the equatorin gene-deleted mouse line has been established, and the analyses are in progress in our laboratory. Also, to visualize the behavior of equatorin, we are performing varieties of imaging studies employing Eqtn-EGFP transgenic mouse spermatozoa. 


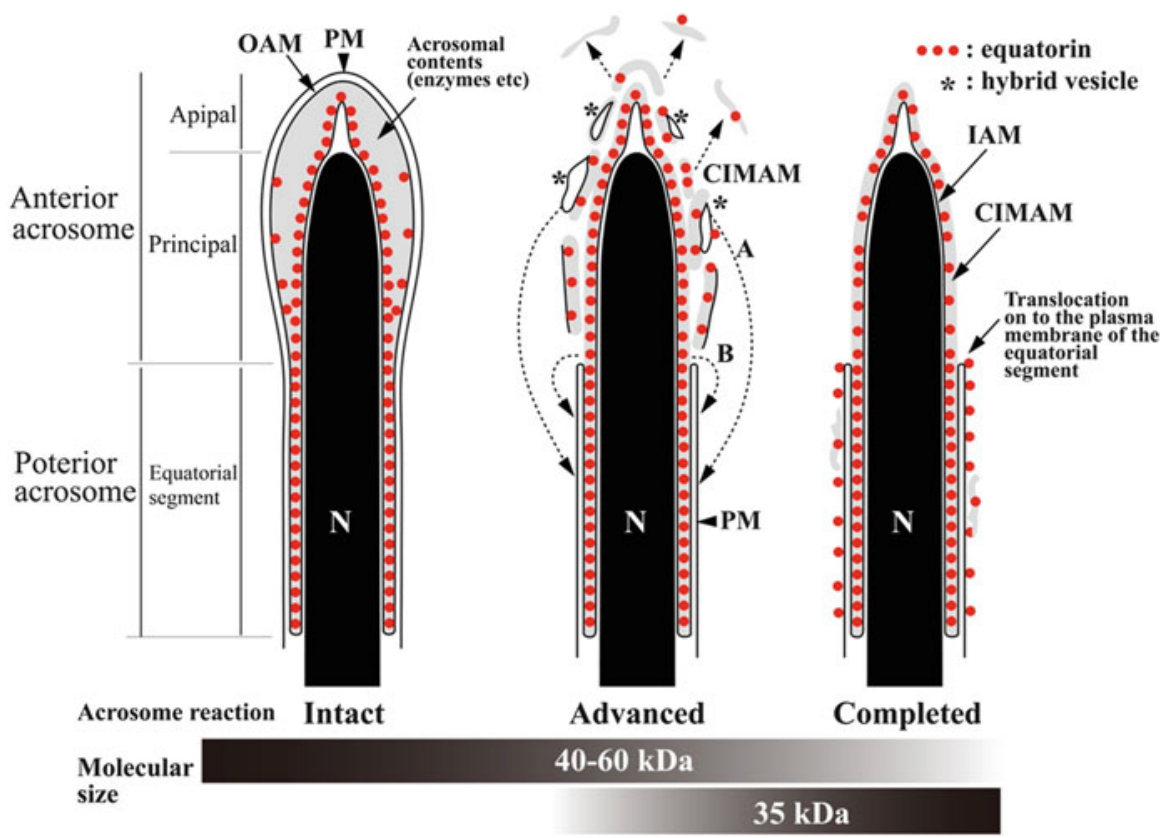

Fig. 7.5 Change of the localization and molecular size of equatorin during the acrosome reaction. Before the acrosome reaction (intact), equatorin (red) is enriched on the whole inner acrosomal membrane $(I A M)$ and the outer acrosomal membrane $(O A M)$ at the equatorial segment but is absent in the apical region and sparse on the outer acrosomal membrane in the principal region. There are several possible routes for the relocation of equatorin onto the sperm surface. First, according to the reassociation model indicated in $\mathbf{A}$, as the acrosome reaction proceeds, equatorin (*) detaches from OAM in the principal region or from other acrosomal membranes and reassociates with the sperm surface; a sufficient amount of equatorin remains associated with the amorphous substance of the acrosomal matrix (gray color). Second, according to the lateral diffusion model indicated in $\mathbf{B}$, equatorin in the acrosomal lumen diffuses to the sperm surface along the acrosomal membrane and plasma membranes $(P M)$, and some of the equatorin becomes localized at the plasma membrane over the equatorial segment $(E S)$; a sufficient amount of equatorin remains on IAM in acrosome reaction-completed spermatozoa. As the acrosome reaction progresses, low molecular mass equatorin $(35 \mathrm{kDa})$ appears and increases in signal intensity. $N$ nucleus

Acknowledgments The authors thank Ms. K. Kamimura and Mr. T. Mutoh for their excellent technical assistance. This work was supported by a grant from the Grant-in-Aid for Scientific Research on Innovation Areas to K.T. (22112504, 24112706), the Grant-in-Aid for Scientific Research to K.T. (22390033), in part to C.I. (24592441) and to K.Y. (23791809).

Open Access: This article is distributed under the terms of the Creative Commons Attribution Noncommercial License which permits any noncommercial use, distribution, and reproduction in any medium, provided the original author(s) and source are credited. 


\section{References}

Bennett EP, Hassan H, Clausen H (1996) cDNA cloning and expression of a novel human UDP-Nacetyl-alpha-D-galactosamine. Polypeptide $N$-acetylgalactosaminyltransferase, GalNAc-t3. J Biol Chem 271:17006-17012

Buffone MG, Ijiri TW, Cao W et al (2012) Heads or tails? Structural events and molecular mechanisms that promote mammalian sperm acrosomal exocytosis and motility. Mol Reprod Dev 79:4-18

Eddy EM (2006) The spermatozoon. In: Knobil E, Neill JD (eds) The physiology of reproduction, vol 1. Raven, New York, pp 3-54

Fawcett DW (1975) The mammalian spermatozoon. Dev Biol 44:394-436

Ferrer M, Rodriguez H, Zara L et al (2012) MMP2 and acrosin are major proteinases associated with the inner acrosomal membrane and may cooperate in sperm penetration of the zona pellucida during fertilization. Cell Tissue Res 349:881-895

Forsbach A, Heinlein UA (1998) Intratesticular distribution of cyritestin, a protein involved in gamete interaction. J Exp Biol 201:861-867

Fujihara Y, Murakami M, Inoue N et al (2010) Sperm equatorial segment protein 1, SPESP1, is required for fully fertile sperm in mouse. J Cell Sci 123:1531-1536

Fujihara Y, Satouh Y, Inoue N et al (2012) SPACA1-deficient male mice are infertile with abnormally shaped sperm heads reminiscent of globozoospermia. Development (Camb) 139: 3583-3589

Hao Z, Wolkowicz MJ, Shetty J et al (2002) SAMP32, a testis-specific, isoantigenic sperm acrosomal membrane-associated protein. Biol Reprod 66:735-744

Inoue N, Ikawa M, Isotani A et al (2005) The immunoglobulin superfamily protein Izumo is required for sperm to fuse with eggs. Nature (Lond) 434:234-238

Ito C, Yamatoya K, Yoshida K et al (2013) Integration of the mouse sperm fertilization-related protein equatorin into the acrosome during spermatogenesis as revealed by super-resolution and immunoelectron microscopy. Cell Tissue Res 352:739-750. doi:10.1007/s00441-00013-01605-y

Kaji K, Oda S, Shikano T et al (2000) The gamete fusion process is defective in eggs of Cd9deficient mice. Nat Genet 24:279-282

Le Naour F, Rubinstein E, Jasmin C et al (2000) Severely reduced female fertility in CD9-deficient mice. Science 287:319-321

Li YC, Hu XQ, Zhang KY et al (2006) Afaf, a novel vesicle membrane protein, is related to acrosome formation in murine testis. FEBS Lett 580:4266-4273

Manandhar G, Toshimori K (2003) Fate of postacrosomal perinuclear theca recognized by monoclonal antibody MN13 after sperm head microinjection and its role in oocyte activation in mice. Biol Reprod 68:655-663

Michaut M, De Blas G, Tomes CN et al (2001) Synaptotagmin VI participates in the acrosome reaction of human spermatozoa. Dev Biol 235:521-529

Miyado K, Yamada G, Yamada S et al (2000) Requirement of CD9 on the egg plasma membrane for fertilization. Science 287:321-324

Miyazaki T, Mori M, Yoshida CA et al (2013) Galnt3 deficiency disrupts acrosome formation and leads to oligoasthenoteratozoospermia. Histochem Cell Biol 139:339-354

Okabe M, Adachi T, Takada K et al (1987) Capacitation-related changes in antigen distribution on mouse sperm heads and its relation to fertilization rate in vitro. J Reprod Immunol 11:91-100

Ruiz A, Pujana MA, Estivill X (2000) Isolation and characterisation of a novel human gene (C9orf11) on chromosome 9p21, a region frequently deleted in human cancer. Biochim Biophys Acta 1517:128-134

Satouh Y, Inoue N, Ikawa M et al (2012) Visualization of the moment of mouse sperm-egg fusion and dynamic localization of IZUMO1. J Cell Sci 125:4985-4990

Shetty J, Wolkowicz MJ, Digilio LC et al (2003) SAMP14, a novel, acrosomal membrane-associated, glycosylphosphatidylinositol-anchored member of the Ly-6/urokinase-type plasminogen activator receptor superfamily with a role in sperm-egg interaction. J Biol Chem 278:30506-30515 
Tomes CN, Michaut M, De Blas G et al (2002) SNARE complex assembly is required for human sperm acrosome reaction. Dev Biol 243:326-338

Toshimori K (2009) Dynamics of the mammalian sperm head: modifications and maturation events from spermatogenesis to egg activation. Adv Anat Embryol Cell Biol 204:5-94

Toshimori K (2011) Dynamics of the mammalian sperm membrane modification leading to fertilization: a cytological study. J Electron Microsc (Tokyo) 60(Suppl 1):S31-S42

Toshimori K, Tanii I, Araki S et al (1992) Characterization of the antigen recognized by a monoclonal antibody MN9: unique transport pathway to the equatorial segment of sperm head during spermiogenesis. Cell Tissue Res 270:459-468

Toshimori K, Saxena DK, Tanii I et al (1998) An MN9 antigenic molecule, equatorin, is required for successful sperm-oocyte fusion in mice. Biol Reprod 59:22-29

Wolkowicz MJ, Shetty J, Westbrook A et al (2003) Equatorial segment protein defines a discrete acrosomal subcompartment persisting throughout acrosomal biogenesis. Biol Reprod 69: 735-745

Wolkowicz MJ, Digilio L, Klotz K et al (2008) Equatorial segment protein (ESP) is a human alloantigen involved in sperm-egg binding and fusion. J Androl 29:272-282

Yamatoya K, Yoshida K, Ito C et al (2009) Equatorin: identification and characterization of the epitope of the MN9 antibody in the mouse. Biol Reprod 81:889-897

Yanagimachi R (1994) Fertilization. In: Knobil E, Neill JD (eds) The physiology of reproduction, vol 1. Raven, New York, pp 189-317

Yoshida K, Ito C, Yamatoya K et al (2010) A model of the acrosome reaction progression via the acrosomal membrane-anchored protein equatorin. Reproduction 139:533-544

Yoshinaga K, Tanii I, Oh-oka T et al (2001) Changes in distribution and molecular weight of the acrosomal protein acrin2 (MC41) during guinea pig spermiogenesis and epididymal maturation. Cell Tissue Res 303:253-261

Yu Y, Xu W, Yi YJ et al (2006) The extracellular protein coat of the inner acrosomal membrane is involved in zona pellucida binding and penetration during fertilization: characterization of its most prominent polypeptide (IAM38). Dev Biol 290:32-43

Yunes R, Tomes C, Michaut M et al (2002) Rab3A and calmodulin regulate acrosomal exocytosis by mechanisms that do not require a direct interaction. FEBS Lett 525:126-130 